\title{
El podcast como herramienta para la innovación en espacios de comunicación universitarios*
}

\author{
Boris Quintana Guerrero** \\ Carolina Parra Duque*** \\ Johanna Paola Riaño Peña ${ }^{* * * *}$ \\ Recibido: 2016-08-21 Enviado a pares: 2016-08-23 \\ Aprobado por pares: 2016-09-06 Aceptado: 2016-11-11 \\ DOI: 10.22395/angr.v15n30a4
}

\section{Resumen}

Este artículo hace referencia a un proyecto de investigación en el que, posterior a un análisis del estado del arte colombiano de la radio universitaria y sus dinámicas de difusión a través de Internet, se seleccionó el podcast como catalizador de formulación y construcción de un laboratorio (RadioLAB) para la elaboración de contenidos de audio digital y su divulgación en el interior del programa de Diseño Industrial de la Fundación Universidad Autónoma de Colombia (FUAC). En una fase de pre-test en un grupo de estudiantes y posterior a ejecutar un estímulo en un momento pos-test, se analiza la percepción de los mismos al implementar podcasts en sus hábitos de estudio; adicionalmente, se identifica la recepción de algunas de las principales directivas de la universidad incluyendo su rector, en cuanto a una posible construcción de un laboratorio de difusión donde tradicionalmente no se cuenta con canales de divulgación (broadcasting). Desde la academia, la implementación de formatos digitales sonoros ofrece grandes ventajas al contemplarse como herramientas de estudio y ser involucrados por docentes en sus clases (igualmente visto en investigaciones latinoamericanas), o simplemente como espacios de comunicación y participación extracurricular. En aproximación a este tema se implementó una metodología experimental y se involucraron estudiantes en una dinámica productores-consumidores (prosumidores). A las conclusiones de percepción se suman la puesta en marcha del laboratorio gracias a los agentes que lo permitieron, y una descripción de enlaces institucionales con los que se está fortaleciendo este nuevo espacio.

Palabras clave: radio en Internet, aprendizaje en línea, grabación sonora.

Este artículo es resultado del proyecto de investigación Radio a la carta: Laboratorio para la difusión del pensamiento en diseño, en la línea de investigación Diseño para la Sostenibilidad del grupo de investigación Centro de Estudios Interdisciplinarios para el Desarrollo (CEIDE) proyecto financiado por el Sistema Unificado de Investigaciones (SUI) de la Fundación Universidad Autónoma de Colombia e inscrito en el Programa Académico de Diseño Industrial. COLOMBIA.

.. Diseñador Industrial de la Universidad Nacional de Colombia. Docente, investigador y Director del Centro de Estudios Interdisciplinarios para el Desarrollo -CEIDE- adscrito al programa de Diseño Industrial en la Universidad Autónoma de Colombia. Estudiante de doctorado en Proyectos de la Universidad Internacional Iberoamericana (México); Máster en Cambio Global de la Universidad de Córdoba (España) y especialista en Edumática de la Universidad Autónoma de Colombia. Se ha desempeñado como catedrático de la Universidad Jorge Tadeo Lozano (Colombia), Universidad ISTHMUS (Panamá), como profesor asociado de la Universidad Nacional de Colombia y como diseñador y consultor en organizaciones manufactureras y culturales Fundación Universidad Autónoma de Colombia. Director Centro CEIDE. centro.ceide@gmail.com

... Diseñadora Industrial de la Universidad de los Andes, candidata a magíster en Semiótica en la Universidad Jorge Tadeo Lozano. Investigadora académica del Centro CEIDE en la Universidad Autónoma de Colombia. Se ha desempeñado como investigadora académica en el departamento de Diseño de la Universidad de los Andes. Ha trabajado como gestora de comunidades virtuales y editora de publicaciones web en temas de diseño en DiseñoLA.org. Cuenta con formación complementaria en Artes Plásticas e Historia y Teoría del Arte. Fundación Universidad Autónoma de Colombia. cparracarol@gmail.com

... Diseñadora industrial de la Universidad Autónoma de Colombia, integrante del semillero de investigación académica de egresados del Centro de Estudios Interdisciplinarios para el Desarrollo -CEIDE-. Ha trabajado en la gestión y fomento al consumo de caucho natural colombiano mediante la formulación de nuevos productos industriales. Participante en la Agenda Prospectiva de Investigación Cadena del Caucho (2012), del Instituto Colombiano Agropecuario -ICA-, y la Agenda de investigación del caucho natural y su industria (2012) de la Cámara de Comercio de VillavicencioFundación Universidad Autónoma de Colombia. johannariano.ds@gmail.com 


\title{
The podcast as a tool for innovation in university communication spaces
}

\begin{abstract}
This article refers to a research project that analyzes the Colombian state of the art in university radio and its Internet broadcast dynamics. The podcast was selected as an issue to develop a laboratory (RadioLAB) for the construction of digital audio contents and its broadcast within the Industrial Design Degree Program of the Fundación Universitaria Autónoma de Colombia (FUAC, an institution where broadcast platforms does not exist). The study make emphasis on a pre-test phase in a group of students, a stimulus and a post-test that evidenced the perception of students by implementing podcast in their study habits; additionally the research group received some guidance for construction of a laboratory by some university directors. The implementation of sound digital formats offers great advantages as a study tools; this research invites teachers to involve this subject in their classrooms. The study approaches the podcast issue by an experimental methodology, where students were used as producers and consumers (prosumers). We add to the conclusions about stakeholder's perception, the launch of the lab through agents who allowed to this construction, and a description of the link with some institutions that strengthens the RadioLAB.
\end{abstract}

Key words: Internet radio, online learning, sound recording.

\section{O podcast como ferramenta para a inovação nos espaços universitários de comunicação}

\begin{abstract}
Resumo
O presente artigo refere a um projeto de pesquisa no qual, após analisar o estado da arte na Colômbia no que diz respeito à rádio universitária e às suas dinâmicas de difusão pela Internet, apontou o podcast como catalizador para a formulação de um laboratório radial. 0 RadioLAB visa a construção de conteúdos de áudio digital, bem como a divulgação desses ao interior do curso de graduação em Design Industrial da Fundación Universitaria Autónoma de Colombia (FUAC). Durante uma fase prévia ao teste com um grupo de estudantes e, após a momento pós-teste, analisa-se a percepção deles ao incluírem o podcast nos seus hábitos de estudo. Além disso, identifica-se a recepção de alguns diretivos da Universidade, inclusive seu reitor, enxergando a possível construção de um laboratório de difusão, diante da carência de canais de divulgação (broadcasting). Da academia, a implementação de formatos digitais sonoros oferece grandes vantagens, ao serem entendidos como ferramentas de estudo quando empregados pelos docentes nas suas aulas (fato salientado pela pesquisa, bem como em outras investigações latino-americanas). Esses formatos também aparecem como simples espaços de comunicação e participação alternativa às atividades curriculares. Para debruçar no tema, lançou-se mão de uma metodologia experimental, convidando estudantes para criar uma dinâmica produtores-consumidores (prosumidores). Às conclusões da percepção, seguem o início de atividades do laboratório, graças aos agentes que o viabilizaram, e uma descrição de vínculos institucionais que contribuem ao fortalecimento deste novo espaço.

Palavras chave: rádio na Internet, aprendizagem on-line, gravação de som
\end{abstract}




\section{Introducción}

Con el creciente número de usuarios de Internet móvil, así como en la diversidad de formatos disponibles en los nuevos dispositivos electrónicos personales que permiten el acceso a redes de Internet, se ha venido cambiando la estrategia de la oferta radial de la radiofrecuencia a la radio basada en la World Wide Web. Esta transición de la tecnología analógica a la digital tuvo sus inicios en los años noventa y cambió de manera significativa a la radio tradicional en aspectos de producción, difusión y recepción; de igual manera permitió el enriquecimiento del audio como medio esencial de la radio, a un medio beneficiado por la convergencia multimedia generando lo que hoy se conoce como ciber radio, que modificó el concepto tradicional de radio a través de una nueva fórmula de programación fragmentada "que sustituye a la oferta de programas de emisión única y continuada, y audición masiva" (González, 2010, p. 53). Esta multimedia permite aprovechar una gran cantidad de información en diferentes formatos entre los cuales se destaca el podcast o radio a la carta, el cual no necesita de un programa radial "al aire" para su emisión. El podcast puede usarse con diferentes propósitos sonoros y, a su vez, permite la búsqueda, escucha y descarga asincrónica a través de la web por medio de dispositivos móviles; el tipo de descarga no constituye una limitante para el acceso a los productos radiales debido a las ventajas del ancho de banda con el que cuentan el Internet móvil, el doméstico y el institucional en nuestros días.

El nacimiento y desarrollo de la ciber radio ha ampliado las posibilidades a instituciones de Educación Superior, remontando de lejos las expectativas iniciales o llevándolas a ostentar, como en el caso de la radio Altair de la Universidad de Antioquia, posiciones privilegiadas dentro de las emisoras virtuales en Latinoamérica, como anota Daniel Urrea editor de Contenidos Hipermediales de Altair, (citado por Ministerio de Educación Nacional, 2007) "no es una señal de radio análoga en la web, sino que son contenidos creados especialmente para la Internet, teniendo en cuenta todo lo que eso conlleva" ( $p$. 1). Al nivel nacional, según la Red de Radio Universitaria de Colombia' (2014) las emisoras universitarias vienen consolidándose como un medio de difusión dinámico que involucra diferentes actores entre estudiantes, docentes, comunicadores, representantes institucionales y público en general en un conglomerado que actualmente comprende alrededor de 63 emisoras, 44 universidades y 20 ciudades.

Una primera aproximación a estas dinámicas de ciber radio y del contexto universitario permite entender que es latente una oportunidad de investigación asociada a la Fundación Universidad Autónoma de Colombia, que actualmente carece de un medio de difusión sonora que facilite su trabajo institucional en términos de comunicaciones e intercambio con el exterior, dinamice los procesos de docencia, formación y extensión, potencie eventualmente los resultados de investigación, enlace e identifique a su comu-

1 La RRUC es la Red de Radio Universitaria de Colombia y define: "Las emisoras educativas universitarias tienen a su cargo la transmisión de programas de interés cultural, sin ninguna finalidad de lucro, con el objeto, entre otros, de difundir la cultura, la ciencia y la educación, de estimular el flujo de investigaciones y de información científica y tecnológica aplicada al desarrollo". Recuperado de https://sites.google.com/a/ radiouniversitaria.org/rruc/ el 03 de febrero de 2015. 
nidad, y fomente, en últimas, su desarrollo cultural, artístico e intelectual. De acuerdo con lo anterior, surgió la oportunidad para desarrollar un laboratorio de podcasting como espacio de comunicación para ser implementado en la FUAC, buscando una sensibilización hacia la comunidad universitaria, a fin de acoger un instrumento más de comunicación en aras del adecuado funcionamiento de esta IES. Por esta razón este proyecto abre una puerta para el posible desarrollo y consolidación de una futura radio web universitaria diseñada e instalada a su escala.

Esta investigación se centró en el formato podcast y en el desarrollo de contenidos con soporte online de carácter descargable que permiten ampliar el espectro de información aplicable a uno o varios programas académicos en función de la ampliación de sus canales de distribución y contacto extracurricular con sus estudiantes. Su justificación estuvo basada en cuatro categorías generales en torno a la conveniencia de poner en marcha una iniciativa de este tipo en la FUAC debido a que este medio se convierte en una plataforma de creación, expresión, integración y mediación: como plataforma de creación, a través de un espacio de laboratorio en función de la exploración de nuevos formatos y estéticas de expresión que permiten la exposición de ideas, se contribuye a la creación de nuevos contenidos y al desarrollo de habilidades por parte de los estudiantes, mejorando su desempeño en la oralidad y fortaleciendo el sentido crítico en expositores y escuchas; una plataforma de expresión que permita el anuncio y promoción de actividades de investigación por parte de estudiantes, docentes, investigadores y egresados de la universidad. Asimismo, la expresión de saberes desde múltiples disciplinas, el arte y la cultura para ser difundidos en aras de transferir el conocimiento a la sociedad; actúa como plataforma de integración al empoderarse de la gestión de identidad local porque facilita la integración de los individuos para encontrarse, identificarse y pensarse como colectivo, permitiendo la opinión de actores administrativos y estudiantiles en una dinámica de comunicación en función de la construcción de comunidad; finalmente se establece como plataforma de mediación entre la universidad y la sociedad dada la necesidad de generar lazos de cooperación y de confianza entre esta y sectores productivos, administrativos nacionales, regionales y distritales, al igual que empresas, asociaciones, agremiaciones y otras comunidades a fin de lograr la ampliación de escenarios de acción que permitan su desarrollo.

Un proyecto de difusión radial tiene múltiples aristas de expansión, y un ejemplo fehaciente de las potencialidades de involucrar iniciativas radiales a los establecimientos educativos se presentó con la implementación del proyecto Estrategia de Radio Educativa, promovido por la Oficina de Innovación Educativa con Uso de Nuevas Tecnologías del Ministerio de Educación Nacional en 2014, y que cuenta como principal aliado a Señal Colombia-Sistema de Medios Públicos. El rápido crecimiento de las tecnologías de distribución determina, a su vez, el avance en la oferta de contenidos haciendo posible la especialización de programas ofrecidos desde Internet; es por esto que el ciberespacio se convierte en una plataforma adaptable para la difusión de contenidos académicos desde la visión particular del pensamiento en diseño, debido a su versatilidad en cuanto a herramientas de producción, y uso de software y hardware a bajo costo. Es necesario 
recordar que la dinámica actual de la radio universitaria no es ajena a estos fenómenos y su propósito es prestar un servicio a la comunidad, apuntando a sectores específicos de la población, enfocado particularmente en el público juvenil, en donde "su principal objetivo es efectuar labores de apoyo a la cultura, la orientación, la enseñanza y la difusión de la investigación científica y humanística" (Zambrano, 2012. p. 124).

Según el estudio preliminar de estas dinámicas de comunicación, se despertó la inquietud en el grupo de investigación acerca de la manera adecuada para la difusión de contenidos académicos en la web, para lo cual se estableció una pregunta orientadora para el presente proyecto: ¿Es posible establecer un medio tipo broadcast con función académica al servicio de la Fundación Universidad Autónoma de Colombia? Y en caso de responder afirmativamente ¿cómo se podría implementar en la universidad? En respuesta a lo anterior se formuló ante el Sistema Unificado de Investigaciones (SUI), el proyecto de investigación Radio a la Carta, Laboratorio para la difusión del pensamiento en Diseño, el cual fue aceptado y financiado por esta IES. En este trabajo se estableció como objetivo principal la introducción a la comunidad académica de la FUAC en el desarrollo e implementación de un laboratorio de difusión de contenidos sonoros como un medio integrador de saberes que permita la ampliación de sus espacios académicos, de vida universitaria y de integración con la sociedad, mediante diversas etapas proyectuales para su exploración, experimentación y validación de su pertinencia. Asimismo, se establecieron como objetivos específicos: (1) Ampliar información contextual de la radio universitaria en Colombia y en el exterior a fin de encontrar actores externos para generar nexos y que fortalezcan el proyecto en la FUAC; (2) Validar al nivel institucional de la Fundación Universidad Autónoma de Colombia la necesidad de conceptualizar, diseñar e implementar una plataforma de podcasting determinada como una etapa piloto; (3) Crear un laboratorio de bajo presupuesto para la investigación, diseño y producción de contenidos para ser ofrecidos al programa académico de Diseño Industrial mediante un servicio de ciber radio en la categoría de podcasting. (4) Desarrollar en una primera etapa una experimentación en podcasting involucrando diversas temáticas en diseño las cuales podrán ser aprovechadas por los estudiantes en pregrado y posgrado.

\section{Marco teórico}

Este trabajo consolidó el desarrollo de un marco teórico referencial con respecto al tema general de la radio universitaria, y en particular de las dinámicas actuales de la radio basada en Internet.

En primera instancia se puede afirmar que la radio ha logrado situar la voz en el primer lugar del diálogo, el discurso y la libertad de pensamiento, que constituyen, a su vez, los principales elementos que discurren alrededor de la construcción del conocimiento en el ámbito universitario. Para su uso y difusión se pueden definir herramientas como las emisoras educativas las cuales son las encargadas de divulgar principalmente la cultura, la música, y la trasmisión de programas especializados de divulgación científica como lo señala la RRUC (2014), generando en su audiencia una aproximación a los avances tecno- 
lógicos, sin olvidar su función social en formación ciudadana y fomento de la inclusión y los espacios para la convivencia.

Desde el punto de vista del crecimiento de la radio universitaria y su impacto al nivel regional, en Colombia se tiene como referente inicial la radio de la Universidad de Antioquia creada en 1933, resultado del trabajo de los profesores José J. Sierra y Próspero Ruiz, quienes en aquel año conformaron una emisora casera de onda corta con una banda de 62 metros; pero su intento traspasó las fronteras nacionales y ahora llega a más de 18 países, como comentan Mesa y Vargas (2014). Aunque ese fue solo el comienzo de la trayectoria de esta radio universitaria, en 2005 logró la creación del Sistema de Radio Educativa, que contó con el apoyo de diferentes instituciones gubernamentales², y se estableció desde entonces como política regional para el fortalecimiento de la educación, brindando nuevas vías de acceso a la información, a la educación formal, a la no formal y a la cultura en la región antioqueña.

Algunas emisoras universitarias se convierten en una alternativa en el medio radial frente a los grandes medios económicos que dominan la franja en la banda de FM, ya que en su parrilla de programación se incluyen programas especializados en géneros musicales, contenidos académicos y de información cultural, y a través de su página web se puede acceder a diversos contenidos en podcast, así como a su señal en vivo. En contraste, la audiencia de la radio de radiofrecuencia está orientada a grandes grupos y comunidades por medio de contenidos generales y comerciales, mientras que la radio en línea se puede enfocar en pequeños grupos de personas con intereses muy particulares y comunes llegando a públicos especializados ya que "permite al oyente construir su programación en función de sus gustos o demandas informativas". (Salgado, 2011, p. 4). Las posibilidades de interactuar asincrónicamente con la radio web permiten al usuario seleccionar por demanda sus contenidos en un tiempo "personal", un tiempo asincrónico a la etapa de producción de cualquiera de estos lo cual permite que el uso de la radio web sea autónomo e interactivo.

Hasta el momento se ha hablado indistintamente de ciber radio, radio por Internet, denominada también como radio virtual, radio web, entre otros, pero existen algunas diferencias entre las emisoras basadas en la web, importantes de entender, como las tipologías señaladas por Rivadeneyra (2013) como son: a) radio por Internet, b) radio web (radio on line), c) podcasting y d) reproductores on line. La radio por Internet fue la respuesta al uso de la web por parte de las emisoras convencionales basadas en transmisión terrestre; así, la señal en vivo pasaría a convertirse en una señal en línea por las condiciones sincrónicas propias de esta radio; de este modo Internet funcionaría como una "antena de repetición", la red re-difundiría la señal que se produce en cualquier emisora convencional, y amplía la audiencia por su gran poder de difusión. La radio web o la radio on line son emisoras que se ofrecen exclusivamente en Internet, las cuales, en la mayoría de los casos, desa-

2 Ejemplo de dichas instituciones son: la Secretaría de Educación para la Cultura, el Instituto para el Desarrollo de Antioquia y la Escuela de Gobierno y Políticas Públicas de Antioquia Guillermo Gaviria C., según lo expresado por Mesa y Vargas (2014). 
rrollan su programación en vivo, aunque ofrecen la posibilidad de acceder posteriormente a algunos de los programas más importantes de su parrilla de programación. La tercera tipología es el podcasting que se perfila como una de las herramientas más versátiles y novedosas por ofrecer grandes cantidades de información a públicos muy heterogéneos, recapitulados en vastas colecciones virtuales. Por último, los reproductores online y las comunidades musicales tienen como protagonista al prosumidor, quien es aquel "consumidor que se implica de forma activa en el desarrollo de productos" (Sheehan, 2012, p. 23), donde se logra la interacción entre quienes producen música y la ofertan, consumen, califican, etc. Todas estas modalidades han generado una renovación en los modelos de negocio y estrategias comerciales de crecimiento para la industria del entretenimiento a través del ciberespacio.

Dentro de estas dinámicas es importante tener en cuenta el concepto de podcast que trata de un conjunto de archivos alojados en un servidor al que es posible suscribirse para recibir todas las actualizaciones posibles sobre los mismos, así en el evento que se vayan presentando se recibirán las actualizaciones en el dispositivo personal conectado a la world wide web. Es un contenido sonoro grabado, el cual puede ser consultado a la carta en cualquier momento luego de su emisión o alojamiento en el servidor, y tiene dos tipos de presentaciones básicas como son el audio y el video, los tipos de archivos mp3 y ACC, para la primera categoría de presentación y mov, m4V y mp4, para la segunda.

En cuanto a la expresión podcast, según Safko \& Brake (2009), proviene de la combinación de términos "iPod y broadcast", aunque también se afirma que el término "podcasting" surge de la combinación de "public on demand" y "cast", y es una transmisión pública que se descarga según demanda. La publicación de podcast típicamente es parte de un blog estructurado por una plantilla en orden cronológico, que muestra las publicaciones realizadas; por tanto, la publicación vista de manera sintética es la producción de audio0/ vídeo, que se debe subir a un servidor web el cual lo aloja (Gardner Campbell, 2005).

Para la producción de podcasts es necesario contar con una serie limitada de implementos en los que no se discutirá técnicamente cada uno por no ser objeto del presente artículo; simplemente se quiere anotar que, debido a las importantes prestaciones de los computadores personales en la actualidad, su implementación ha significado una reducción importante de recursos en los espacios de grabación. Hoy un computador se convierte en el cerebro que conjuga los distintos elementos que deben incorporarse en la producción de podcasts. Adicionalmente, se suma al computador una mesa de mezcla (mixer) dentro de la cual viene incorporada, no en todos los modelos, una interfaz para convertir la señal análoga que viene de los micrófonos en una señal digital que llega al equipo por cableado USB. De igual modo no se pueden dejar por fuera los micrófonos, sus bases o stands, sus espumas o filtros de ruido, el cableado respectivo y un sistema de retorno a los usuarios de la cabina de grabación, y para quien produce, consistente en audífonos personales que se enganchan al sistema por medio de un amplificador de audífonos (mini mixer de canales independientes) que se encarga de balancear los volúmenes de los diferentes actores de 
grabación en sala para permitir al productor la mejor mezcla posible. A esto se suma la locación que debe considerar un mínimo nivel de insonorización.

Debido a la creciente oferta de contenidos digitales, la academia se ha fortalecido y ha encontrado en el podcasting una nueva forma de difusión de contenidos a los estudiantes con el servicio de suscripción; ahora quien estudia puede encontrar una nueva manera de recibir información por fuera del aula de clase. Para lograr dicha suscripción solo será necesario contar con un podcatcher o programa de recogida de archivos podcast a fin de obtenerlos. La gran ventaja es que en la actualidad casi todos los dispositivos que se conectan a la red tienen un podcatcher instalado que permite recibir y dar lectura (de audio o vídeo) de los archivos podcast. Con este tipo de recursos, los profesores pueden ampliar sus clases, orientar trabajos e, incluso, abrir espacios alternos a las prácticas docentes tradicionales, y encontrar eco en su público de estudiantes. En un estudio hecho por Contreras y Eguia (2009) acerca de los contenidos de aprendizaje para estudiantes de diseño, se halló que al proporcionar a los alumnos universitarios un acceso a la descarga de contenidos educativos mediante un dispositivo móvil, estos lo adoptaron en un nuevo medio de aprendizaje. Dicha investigación también evidencia que el uso de podcast es de total interés para los estudiantes, dado que se ajusta a las nuevas tecnologías y al aprovechamiento del tiempo: "los estudiantes reconocen al podcast, como vehículo para la revisión de contenidos, así como una alternativa al aprendizaje" (Contreras y Eguia, 2009, p. 144). Dada la disponibilidad de tecnología en manos de los jóvenes, una herramienta como el podcast ofrece nuevas oportunidades a las universidades para ampliar el conocimiento y brindar nuevos soportes de enseñanza a los estudiantes. Como referente podemos citar al Proyecto de Innovación Docente llevado a cabo en la Facultad de Ciencias de la Educación de la Universidad de Granada (España) durante el curso 2008 / 2009, "en el que se utilizan los podcast como propuesta didáctica encaminada a introducir varias de las propuestas del Espacio Europeo de Educación Superior (EEES) en el aula: TIC, trabajo autónomo, tarea colaborativa y tutorización" (Ramos y Caurcel, 2011).

Es importante recordar que uno de los principales objetivos del presente proyecto ha sido involucrarlo al espacio académico con el propósito de ampliar los conocimientos de los estudiantes, para lo cual los podcast pueden ser utilizados como una herramienta de enseñanza y aprendizaje en la universidad, ya que como señalan Fernández y Sánchez (2010), diferentes experiencias en contextos de enseñanza han puesto de manifiesto que los podcast aportan flexibilidad al permitir el acceso a la información sonora desde cualquier dispositivo móvil o fijo, revolucionando el panorama educativo. Se ha encontrado a partir de la investigación sobre la radio y las herramientas digitales, que su gran incidencia en Educación Superior, así como su continua especialización y la capacidad para llegar a nuevos públicos, reafirman que la radio continúa en vigencia, y la exploración de narrativas multimedia constituye su mayor ventaja actual. Una radio enriquecida como la que se establece en línea obliga a una revisión acerca de la ampliación de canales de comunicación y de las audiencias a quienes se dirige la universidad como institución activa dentro de la sociedad. 
Como se nombró anteriormente la radio web o radio on line corresponde a emisoras que se ofrecen exclusivamente en Internet, las cuales en la mayoría de casos desarrollan su programación en vivo. Solo para dar un vistazo a un ejemplo desde un punto de vista local es interesante revisar el protagonismo que le ha dado el Distrito Capital de Bogotá a su radio de cabecera la cual es una radio on line (http://www.dcradio.gov.co/). Otras exploraciones desde un punto de vista de la creación de radio, se han realizado en la Universidad del Valle donde sus propuestas estéticas reconocidas como radio experimental en particular con asomos de arte radial, o radio arte, han llevado a la Emisora Univalle Estéreo a recibir galardones internacionales por su apuesta sobre el trabajo experimental (http:// cms.univalle.edu.co/emisora/). Este tipo de orientaciones rompen las rigideces en cuanto a la estilística radial que ha sido establecida de manera clásica por las primeras radios universitarias. Zambrano (2012) afirma: "47 \% de las emisoras tiene un estilo acartonado y aburrido como si fuera una extensión del conservatorio. Usan mínimos efectos, paisajes sonoros y cortinas; es usual la entonación, y la impostación de la voz carece de elementos sorpresivos y cotidianos" (p. 125), lo que lleva en estos casos al fracaso de quienes producen radio como a la frustración de los radioescuchas que necesitan a todas luces nuevas alternativas estéticas y de formatos de presentación.

\section{Metodología}

Con el fin de obtener una aproximación general al problema identificado, y atendiendo a los objetivos planteados, se abordaron dos tipos de investigación: una exploratoria y otra descriptiva (Hernández, Fernández, y Baptista. 2010). La investigación de tipo exploratorio permitió un acercamiento al tema poco conocido de la radiodifusión en el contexto institucional de la FUAC. Dentro del contexto estudiado este tipo de investigación permitió obtener datos acerca de las necesidades emergentes de la comunidad académica y demás actores institucionales frente a las herramientas digitales que demandan nuevas dinámicas de acción y participación en su población. Dicha aproximación permitirá la consecución del segundo objetivo de investigación que está detrás de la búsqueda de indicios que demuestren la necesidad latente de un sistema de divulgación como el citado a lo largo de este texto.

Por su parte, la investigación de tipo descriptivo, encargada de "analizar e inventariar las características de fenómenos, objetos, problemas de estudio para definir su naturaleza" (Escorcia, 2010, p. 26), permitió evidenciar los aspectos más relevantes sobre la radio web al nivel global, la radio universitaria en Colombia y las dinámicas de creación y difusión de contenidos digitales a través de la web; todo esto en cumplimiento a una aproximación de un estado del arte, tal como lo indica el primer objetivo de trabajo, en función de observar las creaciones materializadas en contextos externos a la IES. A partir de estos dos tipos de investigación se diseñó una metodología general del proyecto compuesta por tres etapas generales, divididas, a su vez, en diferentes fases para el desarrollo de las actividades lo que determinó cada etapa bajo objetivos definidos, acciones proyectadas y productos esperados (ver gráfica 1). 
Durante la primera etapa "Explorar", se desarrollaron actividades a partir de dos ejes principales: (a) Actualizar, con el fin de ampliar el marco teórico-referencial y (b) Evaluar cualitativamente dicha información a partir de una matriz de síntesis. A modo de síntesis, luego de este largo trabajo documentado en el informe técnico de la investigación en mención, se concluyó el fácil acceso a recursos tecnológicos para producción y distribución de contenidos, su uso universal a partir de referentes tan importantes como las herramientas digitales tipo iTunes y a "la transmisión y alojamiento de contenidos sonoros a través del streamcasting y podcasting" (Álvarez y Vásquez, 2015, p. 486).

Con el fin de caracterizar el espacio que se empezaba a modelar y que se denominó como RadioLAB en la Universidad Autónoma de Colombia, se levantó el estado del arte de los diferentes medios de difusión radial existentes al nivel global y local en cuanto a protocolos y herramientas de funcionamiento; con estos datos se diseñó una matriz comparativa de medios de difusión, la cual permitió indagar algunas características básicas del espacio como el acondicionamiento acústico y las especificaciones de la sala de producción y edición. Se indagó cómo debe estar conformado el equipo de trabajo y cómo se conciben las temáticas a tratar según el tipo de emisora y contenidos que trasmiten.

Se pudo concluir en principio que la tecnología radioweb se ajustaba a las necesidades iniciales del proyecto para la realización del espacio piloto del RadioLAB, como laboratorio de podcasting, así como la necesidad de un espacio de experimentación para producción de contenidos digitales de audio que permite una eficiente difusión en masa y es usado por la mayoría de los medios de difusión radiales. Aunque no es objeto de estudio de la investigación, no se deben desconocer algunos elementos estructurales para la grabación de podcasts como los aspectos sobre la narración y algunos recursos que resultan atractivos para el oyente, ya que las buenas historias para la audiencia son el resultado de una buena comunicación de manera vivaz, apasionante, atractiva y que no se desvíe del tema central como lo señalado por Harnett, M. (2010). La estructura de un programa grabado o en directo tiene un inicio, un desenlace y un final que debe ser equilibrado para mantener a la audiencia interesada; la duración debe ser calculada y planteada en el desarrollo del guion, ya que de su control depende que los diferentes momentos de la narración guarden un ritmo adecuado.

En el marco de la Etapa 1 descrita en la metodología general y adelantando trabajo hacia la cobertura del objetivo 4 del proyecto, se persiguió conocer los intereses de la comunidad, realizando una prueba de intervención con un grupo 41 estudiantes de último ciclo (jóvenes entre los 22 y 26 años, 46 \% hombres y $54 \%$ mujeres), con quienes se propuso hacer charlas grabadas de manera espontánea sobre temáticas particulares de su interés en términos de diseño.

En la segunda etapa "Construir-Evaluar-Validar" se desarrollaron tres principales ejes de acción: definir, modelar y construir el modelo de difusión, teniendo en cuenta las temáticas sugeridas por la comunidad y los parámetros de difusión en radio web identificados previamente; para su ejecución se capturó información de dos grupos de personas 
diferenciados en la institución quienes permitieron seguir conceptualizando la primera propuesta a experimentar por medio del RadioLAB.

Gráfica 1. Metodología de proyecto

\section{METODOLOGÍA GENERAL DE PROYECTO}
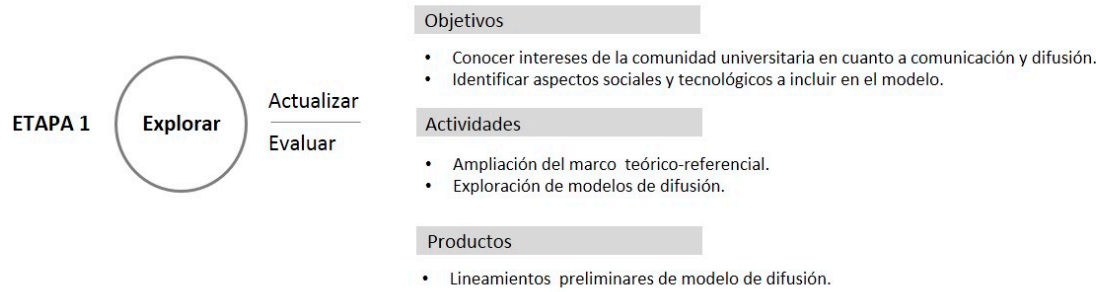

- Conocer intereses de la comunidad universitaria en cuanto a comunicación y difusión.

- Identificar aspectos sociales y tecnológicos a incluir en el modelo.

Actividades

- Ampliación del marco teórico-referencial.

- Exploración de modelos de difusión.

\section{Productos}

- Lineamientos preliminares de modelo de difusión.

\section{Objetivos}

- Caracterizar al público objetivo

- Definir Temáticas y Medios.

Actividades

- Entrevista de diagnóstico.

- Aplicación Diseño de Experimento (pre-test/estimulo/post-test).

Productos

- Evaluación cualitativa de entrevistas y pruebas con grupo objetivo.

- Construcción del modelo de difusión radio web.

\section{Objetivos}

- Difundir el modelo RadioLAB y sus ventajas comunicativas.

- Socializar el modelo con la alta dirección de la universidad y demás actores involucrados.

Actividades

- Implementación de estrategias de difusión del modelo con las partes interesadas.

- Diseño de espacio físico piloto del laboratorio RadioLAB.

Productos

- Desarrollo del espacio físico piloto del RadioLAB.

- Evaluación del modelo RadioLAB implementado y sus ventajas de desarrollo.

Fuente: elaboración propia

El primer grupo correspondió a los directores de las dependencias institucionales, entre los que se cuentan la Rectoría, la Vicerrectoría Académica, el Instituto Superior de Pedagogía (ISP), el Sistema Unificado de Investigaciones (SUI), Bienestar Universitario, la Decanatura de Ingeniería y la Dirección del Programa de Diseño Industrial, con quienes se realizó una entrevista de diagnóstico; los participantes fueron cuatro hombres y tres mujeres adultos, en edades entre los 36 y los 68 años. Se emprendió esta entrevista con el fin de comprender las dinámicas de recepción de los diferentes actores de la alta dirección universitaria en donde se obtuvo una evaluación cualitativa de las expectativas y posibles alcances de un laboratorio radio web en la universidad.

Como resultado de estas entrevistas se reconocieron las incipientes dinámicas de comunicación inter-institucional de las dependencias seleccionadas dentro de la universidad, sus opiniones y posibles necesidades frente a la ampliación e implementación de nuevos canales de comunicación interna y con el exterior; igualmente se buscó sondear preconceptos, expectativas, e ideas por parte de los participantes rumbo a la construcción 
de una nueva plataforma medial de audio digital en la IES. La entrevista fue elaborada a partir de cuatro ejes temáticos, y las respuestas obtenidas están sintetizada en la gráfica 2: (a) descripción de actividades misionales de la dependencia académica; (b) valoración de canales de comunicación; (c) percepción de espacios de difusión audio digital; (d) gestión y apoyo institucional, bajo los cuales se formularon la serie de preguntas que posteriormente encaminaron el análisis y las conclusiones de esta etapa.

Gráfica 2. Resultados Entrevistas

\begin{tabular}{|c|c|c|c|c|}
\hline \multicolumn{5}{|c|}{ EJES TEMÁTICOS } \\
\hline ENTREVISTADO/DEPENDENCIA & $\begin{array}{l}\text { Actividades misionales } \\
\text { dependencia académica }\end{array}$ & $\begin{array}{l}\text { Valoración de canales de } \\
\text { comunicación }\end{array}$ & $\begin{array}{c}\text { Percepción espacios de difusión } \\
\text { audio digital }\end{array}$ & Gestión y apoyo institucional \\
\hline Dirección Universitaria & $\begin{array}{l}\text { Planeación estratégica de la } \\
\text { Organización Dirección General } \\
\text { Universitaria en función de la } \\
\text { calidad educativa }\end{array}$ & $\begin{array}{l}\text { No hay actualmente un sistema } \\
\text { de medios de comunicación } \\
\text { como TV y Radio }\end{array}$ & $\begin{array}{l}\text { Buena iniciativa como } \\
\text { instrumento de divulgación }\end{array}$ & $\begin{array}{l}\text { Avala la pertinencia para la FUAC } \\
\text { del proyecto de investigación }\end{array}$ \\
\hline Dirección Universitaria & $\begin{array}{l}\text { Liderazgo, Apoyo a Rectoría } \\
\text { Acreditación institucional, } \\
\text { internaionalización }\end{array}$ & $\begin{array}{l}\text { Se encuentra en proceso de } \\
\text { actualización de redes internas } \\
\text { de comunicación }\end{array}$ & $\begin{array}{l}\text { Ayuda a la participación de } \\
\text { la comunidad y el trabajo } \\
\text { interdisciplinario }\end{array}$ & $\begin{array}{l}\text { Recomienda revisión de } \\
\text { normatividad de radio web } \\
\text { universitaria }\end{array}$ \\
\hline Pedagogía Universitaria & $\begin{array}{l}\text { Actualización en últimos } \\
\text { desarrollos tecnológicos en } \\
\text { Pedagogía }\end{array}$ & $\begin{array}{l}\text { Carece de un sistema de medios } \\
\text { para que las comunicaciones } \\
\text { internas sean efectivas }\end{array}$ & $\begin{array}{l}\text { Buena iniciativa para la } \\
\text { promoción y difusión de la } \\
\text { comunidad }\end{array}$ & Apoyo desde lo pedagógico \\
\hline Dirección de investigación & $\begin{array}{l}\text { Dirección de procesos de } \\
\text { investigación en la FUAC }\end{array}$ & $\begin{array}{l}\text { Es necesario un medio de } \\
\text { comunicación para la difusión de } \\
\text { la investigación }\end{array}$ & $\begin{array}{l}\text { Buen espacio para la difusión de } \\
\text { actividades académicas }\end{array}$ & $\begin{array}{l}\text { Pertinencia para el } \\
\text { fortalecimiento institucional }\end{array}$ \\
\hline Bienestar universitario & $\begin{array}{l}\text { Atención al estudiante, } \\
\text { promoción y prevención } \\
\text { actividades culturales }\end{array}$ & $\begin{array}{l}\text { Es necesaria la ampliación de } \\
\text { canales de comunicación con } \\
\text { medios multimedia }\end{array}$ & $\begin{array}{l}\text { Facilitar la divulgación de } \\
\text { actividades programadas para la } \\
\text { comunidad }\end{array}$ & $\begin{array}{l}\text { Pautas y contenidos de promoción } \\
\text { en conjunto con comunicación }\end{array}$ \\
\hline Dirección de Facultad & $\begin{array}{l}\text { Dirección de actividades } \\
\text { académicas en Ingenierías, } \\
\text { docencia e investigación }\end{array}$ & $\begin{array}{l}\text { Es necesario tener mayor nivel de } \\
\text { comunicación interna y externa } \\
\text { en la Universidad }\end{array}$ & $\begin{array}{l}\text { Buen camino para implementar } \\
\text { la radio web y la comunicación } \\
\text { interna }\end{array}$ & $\begin{array}{l}\text { Apoyo institucional de la FUACy } \\
\text { sus miembros }\end{array}$ \\
\hline $\begin{array}{l}\text { Dirección de programa } \\
\text { académico }\end{array}$ & $\begin{array}{l}\text { Dirección de actividades } \\
\text { académicas en Diseño Industrial, } \\
\text { docencia e investigación }\end{array}$ & $\begin{array}{l}\text { Se necesita un canal de mayor } \\
\text { difusión de la información }\end{array}$ & $\begin{array}{l}\text { Mejorar la comunicación a } \\
\text { nivel interno y externo de la } \\
\text { Universidad }\end{array}$ & $\begin{array}{l}\text { Apoyo desde el Programa } \\
\text { Académico de Diseño }\end{array}$ \\
\hline
\end{tabular}

Fuente: elaboración propia

Por medio de la entrevista, los participantes de la alta dirección universitaria manifestaron su opinión y percepción según las actividades misionales de cada área al interior de la institución, en donde se evidencia el interés y posible participación en un proyecto de construcción de una plataforma de audio digital en la universidad en apoyo a temas como la educación virtual, el desarrollo de estrategias pedagógicas, difusión de proyectos de investigación, fortalecimiento de la comunidad, promoción y desarrollo humano.

Igualmente, se evidenció la necesidad de implementar canales de comunicación más efectivos en el interior de la universidad entre las dependencias académicas y administrativas, que estén encaminados a fortalecer la institucionalidad de la FUAC. Medios como la radio en Internet, la TV y la aplicación de estrategias multimedia son necesarios para el desarrollo y actualización de la universidad, como lo expresado por Mosquera, R. (2015): "la actualidad obliga a que así como la telefonía móvil es instrumento necesario 
para informarse, la radio puede convertirse en un medio de trabajo". Asimismo, dichos medios constituyen un apoyo importante en procesos como el de acreditación de alta calidad en educación. En cuanto a la creación de espacios para la producción y difusión de contenidos de audio (RadioLAB) para la Universidad Autónoma de Colombia, las distintas dependencias manifestaron un concepto favorable para su creación y sugieren que se desarrolle el proyecto por etapas, con el fin de garantizar su viabilidad a largo plazo; además, que se busquen estrategias de integración entre las facultades académicas y el área administrativa para garantizar el carácter plural y participativo.

El segundo grupo con el que se capturó información correspondió a estudiantes del Programa Académico de Diseño Industrial con quienes se aplicó una prueba (correspondiente a la categoría metodológica del diseño de experimentos) cuyo objetivo fue determinar si los conceptos de este grupo variaban luego de recibir un estímulo (Hernández, et al., 2010). El experimento aplicado se desarrolló en tres etapas: pre-test, estímulo, pos-test, las cuales son definidas y esquematizadas en la gráfica 3.

El grupo de estudio estuvo conformado por discentes del componente microcurricular denominado Diseño de Producto, a quienes se aplicó un pre-test con una herramienta de carácter semi-estructurado que involucró seis puntos básicos que perseguían describir los preconceptos de los estudiantes en términos de conocimiento y uso de herramientas basadas en tecnologías de la información y comunicación (TIC) con referencia a contenidos de audio digital. Específicamente este grupo estuvo constituido por 34 personas de las cuales el 64 \% correspondía a mujeres y 36 \% a hombres, jóvenes entre los 20 y 25 años.

Gráfica 3. Metodología de diseño de experimentos

\section{DISEÑO DE EXPERIMENTO}
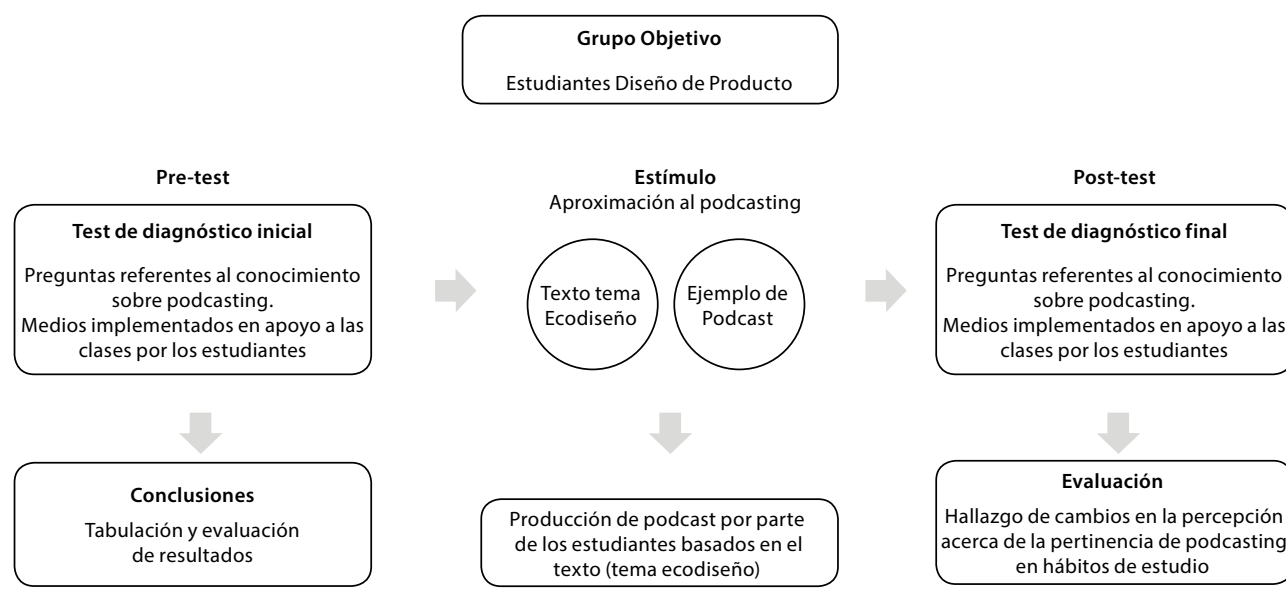

Fuente: elaboración propia 
Por su parte, la aplicación del estímulo consistió en la aproximación al podcasting, entregando un texto al grupo sobre el tema de ecodiseño; adicionalmente se les sugirió escuchar tres archivos de audio (podcast) sobre temas afines, a modo de complemento de la lectura. Este grupo tuvo como tarea desarrollar un debate basado en el texto entregado el cual fue grabado en audio. La finalidad de esta experiencia consistió en que los alumnos ampliaran los límites de asimilación de contenidos que brinda la sola lectura de textos, asunto que permite el podcast profundizar en una temática abordada por medio de otro canal de información. La grabación, a su vez, los introdujo a la producción de podcasts permitiéndoles comprender que su sencilla difusión puede impactar importantemente su medio haciendo uso de sus canales tradicionales de comunicación: las redes sociales; así el podcast empieza a entenderse como herramienta con potencial para dinamizar el proceso de aprendizaje en estudiantes.

Posteriormente a este grupo de estudio se realizó una segunda prueba (pos-test) con el fin de identificar un posible cambio en los preconceptos frente a su percepción en cuanto al uso de contenidos de audio en apoyo a sus actividades académicas.

Luego de realizar la tabulación de datos se procedió a su respectivo análisis. Al terminar la primera prueba (pre-test) se pudo concluir que el uso de otras herramientas y medios de difusión como podcast y blogs, tenía un nivel bajo de implementación por parte de los estudiantes como apoyo a sus clases convencionales, (ver gráfica 4). Esto evidenció inicialmente la falta de conocimiento de los beneficios de dichas herramientas, dado que no se había explorado el uso de las mismas; al respecto los estudiantes manifestaron que no conocían información (en el área del diseño) a partir de los medios citados a fin de mejorar su estudio y / o apoyar sus proyectos académicos. En las respuestas iniciales también se encontró que el correo electrónico (24 \%) y la multimedia (19\%) eran los medios más usados en apoyo a las clases.

Al finalizar la primera prueba se evidenció que, aunque los estudiantes no usaban con frecuencia estas herramientas, sí evidenciaron un interés hacia las mismas para reforzar contenidos recibidos en el aula; manifestaron que herramientas como el blog y podcast les pueden servir como un elemento de ampliación y refuerzo de conocimientos.

Una vez terminada la primera prueba se realizó la aplicación del estímulo en donde se les facilitó a los participantes un texto sobre el tema de ecodiseño para la lectura y posterior discusión en grupo; también se les facilitaron archivos de audio (podcast) para su escucha. A continuación el grupo fue dividido en tres subgrupos, y se realizaron en cada uno de ellos discusiones en torno a la información presentada en lectura y podcasts.

Particularmente y luego de la sesión de discusión con los estudiantes, al aplicar el post-test se identificó que el grupo evaluado logró familiarizarse con el tema; se evidenció una percepción diferente frente al uso del podcast como herramienta, y se encontró algún interés por incorporarla a sus hábitos de estudio, al considerar que posibilita la ampliación de conceptos relacionados con algún tema específico de estudio (ver gráfica 5). En la segunda prueba respondieron de forma afirmativa al uso del blog en un $14 \%$ 


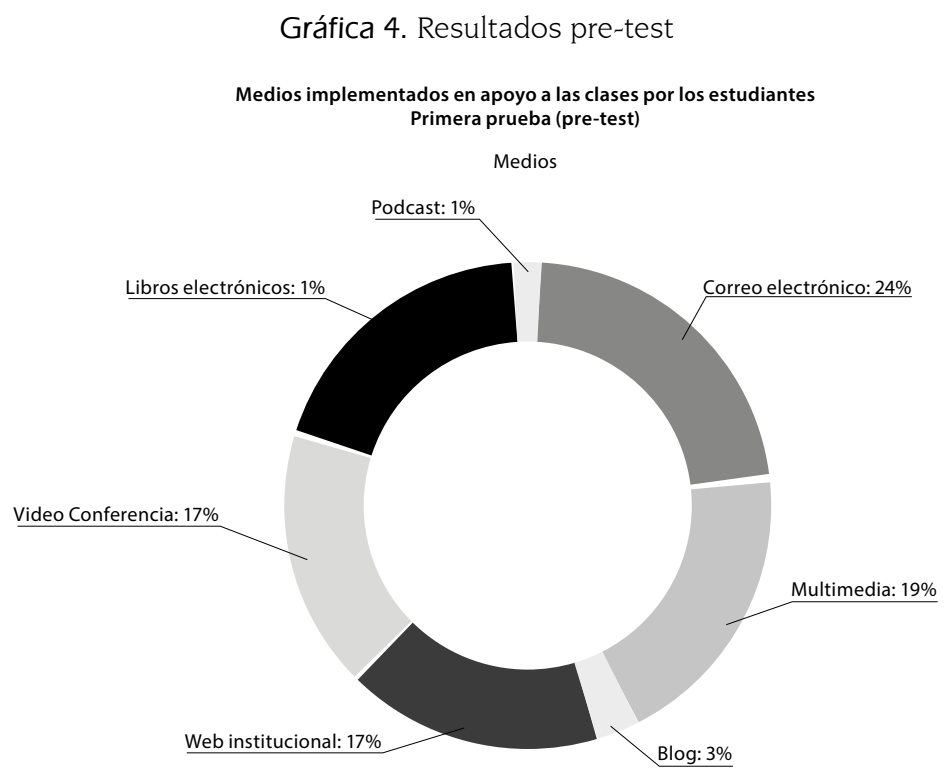

Fuente: elaboración propia

Gráfica 5. Resultados post-test

Medios implementados en apoyo a las clases por los estudiantes Segunda prueba (post-test)

Medios

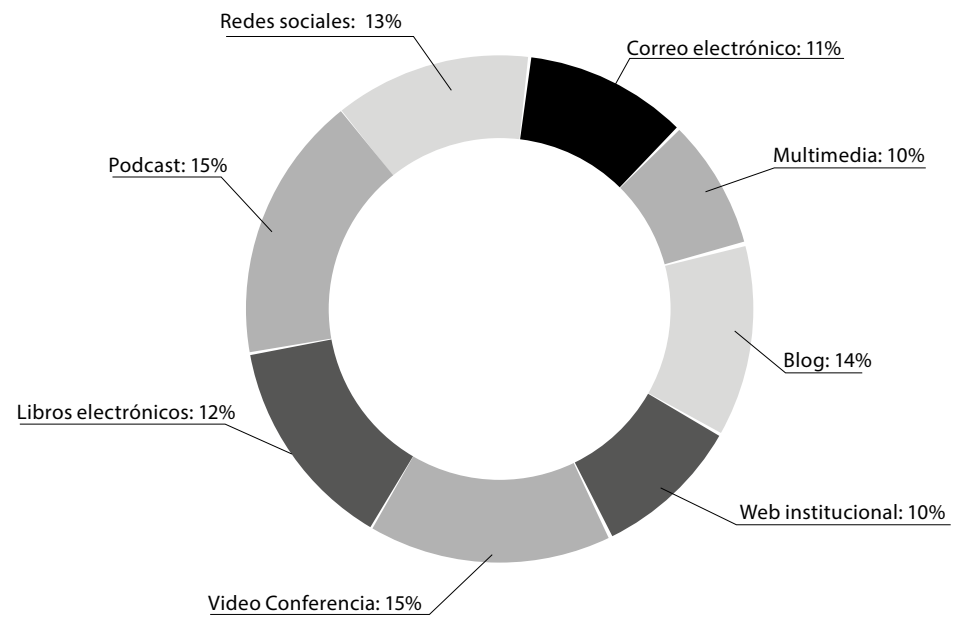

Fuente: elaboración propia 
y del podcast en un $15 \%$; los participantes también sugirieron que el uso de las redes sociales podía ser parte de esta dinámica de contenidos académicos, razón por la cual se incluyó este ítem en la tabulación con un resultado de $13 \%$. El formato podcast puede implementarse progresivamente como un método de enseñanza que mejore tanto la oferta de contenidos como los métodos de estudio, ya que para los estudiantes también resulta interesante la disponibilidad de acceso a la información en repetidas ocasiones a través de la red; además, se observó su interés en volverse agentes activos en la generación y uso de contenidos de audio.

La información capturada durante las entrevistas diagnóstico y las pruebas del diseño de experimentos permitió la generación de lineamientos para el modelo de difusión de podcasting, dado que este debe ajustarse a las necesidades de la comunidad universitaria a fin de poder permanecer y desarrollarse en el tiempo; por tal razón, es pertinente realizar jornadas de socialización sobre la importancia del uso de herramientas web y medios de difusión, haciendo énfasis en el uso de podcast y blogs vía streaming para que los miembros de la comunidad universitaria se familiaricen con estas herramientas. Los contenidos generados deben ser acordes con los temas de las asignaturas del Programa Diseño industrial, a fin de responder a los intereses inmediatos de los estudiantes, y debe surtir bajo dichas expectativas la oferta de podcasts en la propuesta diseñada, en la cual se obtendrán mejores resultados una vez los docentes se incorporen a la misma, y sean quienes articulen temáticamente los podcasts a sus componentes microcurriculares. El modelo debe contar con una estrategia que involucre a los estudiantes tanto en la generación de contenidos, como en la escucha y difusión de los mismos, además de permitirles opinar o realimentar los contenidos publicados.

La tercera y última etapa "Ejecutar" se desarrolló alrededor de dos ejes: describir y socializar un modelo (RadioLAB) que permitiera la producción y difusión de contenidos temáticos desde el área del diseño que fueran de interés para la comunidad universitaria y, asimismo, demostrar las posibilidades y ventajas del desarrollo de un laboratorio de la radio web universitaria para la difusión del pensamiento en diseño.

Según el estudio referencial de la primera etapa, las observaciones recibidas por las directivas de la universidad, y la observación realizada a estudiantes, se perfiló este modelo bajo los siguientes requerimientos generales: a) Difusión: que permita una participación flexible, facilite la disponibilidad de los contenidos en la red y facilite la integración de los diferentes actores de la comunidad universitaria. En este sentido se emplearon redes sociales (Facebook y Twitter) que permanecen conectadas a las actualizaciones efectuadas sobre la plataforma principal (en la cual se alojan y organizan los podcasts). Para su implementación se hizo un estudio de las diferentes plataformas de podcast en el mercado dentro de las cuales se seleccionó la catalana "Ivoox" (www.ivoox.com) b) Desarrollo temático: con el cual se establece una dinámica participativa para el adelanto de contenidos universidad-empresa; este criterio está centrado en el diseño y sus dinámicas interdisciplinares, en donde los temas propuestos a priori corresponden al desarrollo tecnológico, diseño sustentable, diseño y academia, responsabilidad social empresarial, entre otros. c) 
Espacio físico: de acuerdo con los requerimientos necesarios para el diseño e instalación del RadioLAB tales como la acústica, el área de grabación, y la proyección de un espacio que se adapte a las áreas de trabajo requeridas para la producción de un laboratorio de podcasting. En cuanto a esto último vale la pena anotar que la universidad entregó un espacio muerto el cual fue adaptado con estudiantes del semillero de investigación y actualmente se encuentra ubicado como la oficina 504 del bloque de diseño en la sede XX Aniversario de la FUAC. Este espacio fue adaptado por quienes escriben este artículo con la colaboración del semillero de investigación.

\section{Conclusiones}

Basados en la información obtenida de la prueba de grabación en el grupo de 41 estudiantes (de último año de carrera de Diseño), se concluye que más del $90 \%$ de los estudiantes identificaron el uso del podcast como la mejor opción para crear y difundir contenidos sonoros desde un punto de vista de facilidad en la consecución de herramientas para lograrlo, así como la economía en tiempo y dinero que reviste. Dado que el producto se trata de un archivo de audio, este puede ser escuchado y descargado desde la web, puede ser compartido para una vasta audiencia, y permite una amplia difusión al lograrse por medio de redes sociales y herramientas de uso convencional por los estudiantes. El $97 \%$ de los estudiantes se declararon sorprendidos al encontrar que el podcast puede emplearse como un objeto articulable a sus clases, lo cual lo convierte en un elemento promisorio para su implementación académica en la FUAC.

Acorde a la información obtenida en el pre-test descrito en la Segunda Etapa del proyecto, para estudiantes de cuarto año de carrera, el blog y el podcast se muestran como las herramientas de menor uso, 3 y $1 \%$, respectivamente, debido a que no hay suficiente información relacionada con sus necesidades prioritarias desde un punto de vista académico. El afán de los estudiantes por encontrar información inmediata ocasiona falta de rigor en la búsqueda de la misma, y es claro que encontrar un podcast o blog según sus intereses específicos demanda mayor tiempo y esfuerzo.

Luego de la sesión de diálogo y discusión de los estudiantes basado en el texto "Ecodiseño" (estímulo durante la segunda etapa de investigación) y de escuchar los podcast sugeridos, al aplicar el pos-test se identificó que un gran porcentaje del grupo evaluado (75 \%) logró familiarizarse con el tema, lo cual cambió su percepción frente al uso de esta herramienta, y evidenció un interés por hacer uso de este al considerar que posibilita el diálogo y la ampliación conceptos relacionados con un tema en específico en ámbitos de diseño.

Los diferentes aportes de estudiantes y administrativos de la universidad permitieron modelar el RadioLAB, laboratorio de podcasting para la FUAC, caracterizándolo como el modelo de difusión de audio digital a medida actual de la IES; este laboratorio incipiente permitió en el mes de febrero de 2016 preparar una propuesta a la convocatoria de estímulos 2016 del Ministerio de Cultura de Colombia denominado Becas de creación para 
series radiales, franjas de radios ciudadanas-Fortaleciendo una cultura de paz, convocatoria para la que se diseñó y presentó una propuesta de difusión en la que se definió una plataforma que permita la participación activa de los distintos actores involucrados como los estudiantes, docentes, personal administrativo e invitados externos, generando diversas dinámicas de relación entre los mismos en pro de una transformación social escalable. Dicha propuesta trascendió los objetivos del proyecto de investigación al ser incorporados procesos de aprendizaje y reaprendizaje colaborativo, dentro de las dinámicas de educación virtual y el desarrollo de estrategias pedagógicas encaminadas a ser incorporadas en la comunidad en la cual se inscribe.

Durante el proceso de investigación se pudo contar con diferentes entes externos que brindaron aportes en conocimiento y empoderamiento del tema, los cuales permitieron viabilizar de alguna manera la realización de este proyecto; por un lado, se contó con el apoyo del Sistema Radio Candelaria de la Localidad de la Candelaria (localidad donde se encuentra ubicada la FUAC) quienes capacitaron a tres estudiantes del semillero de investigación en el uso de herramientas de planeación y producción de programas radiales; por otro lado, se logró una alianza con la Emisora Comunitaria Suba al Aire 88.4 FM en Bogotá, con quienes se está gestionado un espacio de participación del RadioLAB en su parrilla de programación; dado esto, se sigue trabajando en función de tener un impacto social continuado, es decir, perfeccionar en el tiempo este tipo de alianzas para que diversas poblaciones de la ciudad de Bogotá reconozcan en la FUAC una universidad que trabaja en pro del desarrollo comunitario.

La articulación gestionada entre el grupo investigador, la investigación denominada Radio a la Carta y los diferentes organismos de la dirección de la IES permitió conceptualizar, diseñar y construir el espacio que hoy se reconoce como RadioLAB (https://goo.gl/ aCmiv8) en la Universidad Autónoma de Colombia. Se consolidó como un laboratorio de bajo presupuesto, con vocación para la producción, edición y difusión de podcasting, el cual se encuentra en funcionamiento desde mediados de julio de 2016.

A la fecha se han producido tres episodios (cada episodio es semanal) para el primer programa de variedades en términos del diseño industrial de la FUAC denominado "15 Grados de Diseño". Al igual que este programa, a la fecha algunos docentes preparan contenidos para sus clases y han solicitado la creación de talleres para que tanto ellos como sus estudiantes puedan apropiarse de esta herramienta de la Universidad.

\section{Bibliografía}

Álvarez-Moreno, M. A, Vásquez-Carvajal, S. C. (2015). Radio y cultura: una propuesta de radio ciudadana en Internet. Palabra Clave, Vol. 18(2), 475-498. DOI:10.5294/pacla.2015.18.2.7

Campbell, G. (2005). There`s Something in the Air: Podcasting in Education. EDUCAUSE. Review, Vol. 40 (6), 32-47.

Contreras, R. Eguia, J. (2009). Contenidos de aprendizaje para estudiantes de diseño en podcast. Cuadernos de Documentación Multimedia. Vol. 20, (1), 139-148. 
Escorcia, O. (2010). Manual para la investigación. Guía para la formulación, desarrollo y divulgación de proyectos. Bogotá: Universidad Nacional de Colombia.

González, M. (2010). La ciberradio. Nueva alternativa de futuro para la radio. Revista de Estudios de Juventud, Vol. 88, 51-62.

Harnett, M. (2010). Guía de acceso rápido a podcasting. Cómo crear audio y vídeo para iPods, iPhones, BlackBerrys, móviles y sitios web. Buenos Aires: Ediciones Granica.

Hernández, R. Fernández, C. Baptista, P. (2003). Metodología de la Investigación. México D. F.: McGraw Hill

Mesa, N., Vargas, A. (2014). Perspectiva educativa de la radio universitaria. Estudio de Caso Sistema de Radio Educativa de la Universidad de Antioquia. Revista Fundación Universitaria Luis Amigó. Vol. 1 (1), 42-49.

Ministerio de Educación Nacional (2007) Siete años cumple la primera emisora virtual universitaria de Latinoamérica. Recuperado el 15 de mayo de 2015. Disponible en http://www.mineducacion. gov.co/cvn/1665/article-125315.html

Ministerio de Educación Nacional. Oficina de Innovación Educativa con Uso de Nuevas Tecnologías. (2014). Proyecto estrategia de radio educativa. Recuperado el 15 de mayo del 2015. Disponible en http://www.colombiaaprende.edu.co/html/micrositios/1752/articles338933_archivo_pdf.pdf

Mosquera, R. (25 de noviembre de 2015). Entrevistador S. Gamba [Archivo de audio] Registro de Entrevista diagnóstico para personal académico-administrativo FUAC. Sin publicar.

Ramos, G. Caurcel, C. (2011). Los podcast como herramienta de enseñanza-aprendizaje en la Universidad. Profesorado. Revista de Curriculum y Formación del Profesorado. Vol. 15 (1), 151-162.

Red de Radio Universitaria de Colombia (2014). Radio Universitaria. Principios Organizativos. Recuperado el 03 de febrero de 2015. Disponible en https://sites.google.com/a/radiouniversitaria. org/rruc/Home

Rivadeneyra, C. (2013). La radio en el escenario digital. Escuela de Ciencias de la Información. Comunicación del IV Encuentro Panamericano de Comunicación, Córdoba, Argentina. Recuperado el 15 de mayo de 2015. Disponible en http://www.eci.unc.edu.ar/archivos/companam/ponencias/ Escenarios\%20digitales/-Unlicensed-ESCENARIOS-DIGITALES-Rivadeneyra-vF2.pdf

Salgado, C. (2011). Nuevas narrativas para la ciberradio. Recuperado el 15 de mayo de 2015. Disponible en http://www.gabinetecomunicacionyeducacion.com/sites/default/files/field/adjuntos/nuevas narrativas_para_la_ciberradio.pdf

Safko, L., Brake, D. (2009). The Social Media Bible. Tactics, tools and strategies for business success. New Jersey: Ed.Wiley.

Sheehan, B. (2012). Marketing on-line. Barcelona: Blume.

Zambrano, W. (2012). Radiografía de las emisoras universitarias colombianas. Folios. Facultad de Comunicaciones de la Universidad de Antioquia. (28), 115-138. 
